\title{
Frecuencia de roncopatía en mujeres durante el tercer trimestre del embarazo, en un hospital general.
}

\author{
Snoring frequency in women during the third trimester of pregnancy in a general hospital. \\ Renzo Figari ${ }^{1}$, Angie Nishio ${ }^{2}$, Pedro Saona ${ }^{3}$, DarwinVizcarra ${ }^{4}$.
}

\section{RESUMEN}

Objetivo: Determinar la frecuencia y distribución de la roncopatía durante el tercer trimestre de gestación en pacientes hospitalizadas en un hospital general. Materiales y métodos: Estudio descriptivo y transversal, realizado entre septiembre y octubre del año 2004 en el Hospital Nacional Cayetano Heredia. Se incluyeron 295 voluntarios de 304. Se emplearon 3 encuestas: Cuestionario de Ronquidos, Escala de somnolencia de Epworth y Prueba de Calidad del Sueño. Adicionalmente se aplicó la medida morfométrica de Kushida. Resultados: La edad promedio fue de 25,9 $\pm 1,67$ años; con una edad gestacional promedio de 39,3 $\pm 1,55$ semanas. El 44,7\% presentó roncopatía en el tercer trimestre y el 10,5\% eran roncadoras habituales, $23,7 \%$ reportó tener somnolencia. Solo el $0,7 \%$ tuvo resultado positivo para la medida morfométrica de Kushida. Se encontró un riesgo incrementado de presentar roncopatía habitual en las pacientes mayores de 25 años. Finalmente, el 13,6\% tuvo Hipertensión Inducida por la Gestación y de estas, 22,5\% eran roncadoras habituales. Estas últimas con un riesgo 3 veces mayor de tener hipertensión inducida por la gestación. Conclusiones: La prevalencia de roncopatía y roncadoras habituales durante el tercer trimestre en pacientes hospitalizadas en el Hospital Nacional Cayetano Heredia es similar a la reportada en literatura internacional. El índice de Kushida no resultó aplicable en nuestra población. Existe una relación entre la presencia de roncopatía habitual e hipertensión inducida por la gestación.(Rev Med Hered 2010;21:91-96).

PALABRAS CLAVE: Prevalencia, embarazo, ronquidos, apnea del sueño obstructiva, hipertensión inducida por la gestación.

\section{SUMMARY}

Objective: To determine the frequency and distribution of snoring during the third trimester of pregnancy in patients hospitalized in a general hospital. Material and Methods: We conducted a cross sectional and descriptive study

1 Miembro de la American Academy of Neurology. Residente del Departamento de Neurología en Henry Ford Health System, Detroit, MI, USA

2 Miembro de la American Collegues of Physicians. Residente del Departamento de Medicina Interna en Henry Ford Health System, Detroit, MI, USA.

3 Doctor en Medicina. Profesor Principal Departamento de Obstetricia y Ginecología de la Universidad Peruana Cayetano Heredia. Medico Asistente del Departamento de Obstetricia y Ginecología del Hospital Nacional Cayetano Heredia. Lima, Perú.

4 Médico Neurólogo Especialista en Medicina del Sueño. Director de Hypnos, Instituto del Sueño. Profesor Asociado de la Facultad de Medicina Alberto Hurtado de la Universidad Peruana Cayetano Heredia. Lima, Perú. 
during September and October of 2004. 295 volunteers of a total of 304 were included. We used three validated questionnaires: Snoring questionnaire, Epworth Sleepiness Scale, Sleeping Quality Test. Additionally, we used the Kushida's Morphometric Model. Results: The average age was 25,9 \pm 1,67 years old with an average gestational age of 39,3 $\pm 1,55$ weeks. We found $44,7 \%$ patients with snoring during the third trimester; the frequency of habitual snoring was $10,5 \% .23,7 \%$ of patients reported having somnolence. Only $0,7 \%$ of patients had a positive result in the Kushida's morphometric model. There was an increased risk of having habitual snoring in patients older than 25 years old. Finally, 13,6\% had Pregnancy Induced Hypertension with a 22,5\% of them having habitual snoring. Those with habitual snoring had 3 times higher risk of having pregnancy induced hypertension. Conclusions: This study found the same frequency of snoring and habitual snoring as previous studies in pregnant patients during the third trimester. The morphometric model of Kushida is not applicable to our population. There is a relation between habitual snoring and gestational hypertension. (Rev Med Hered 2010;21:91-96).

KEY WORDS: Prevalence, pregnancy, snoring, sleep apnea, obstructive, hypertension pregnancy-induced.

\section{INTRODUCCIÓN}

Lo que conocemos como ronquido no es sino la inspiración ruidosa producida por la vibración de las partes blandas de la orofaringe (1). Se registra en aproximadamente el $20 \%$ de la población adulta, y luego de los 60 años se puede encontrar $60 \%$ de roncópatas en la población masculina y $40 \%$ en la femenina (2). Si bien puede ser benigno, muchas veces es manifestación de patologías del sueño; encontrándose que uno de cada 5 a 7 personas roncópatas sufre del síndrome de apnea obstructiva del sueño (SAOS) (1). Este síndrome, cuya prevalencia aumenta con la edad, esta asociado a somnolencia diurna y alteraciones cardiopulmonares graves prevenibles con tratamiento precoz como el uso de CPAP (del inglés presión positiva continua sobre la vías aérea) (3-6).

Así mismo se ha reportado la presencia de roncopatía en mujeres caucásicas gestantes. Durante este periodo se observa una reducción de las dimensiones de la faringe con hiperemia, hipersecreción y edema de la mucosa respiratoria; aumento de la ventilación junto con una reducción de la capacidad funcional residual y un aumento en la gradiente de oxígeno alveolo-arterial; una disminución total del tiempo de sueño con incremento de los microdespertares, incremento del estadio 1 del sueño no-REM, disminución de los estadios 3 y 4, y disminución del sueño REM. Estas variaciones anátomo-fisiológicas son mediadas por el aumento de los niveles de estrógeno y progesterona unidos al efecto mecánico que ejerce el útero al final del embarazo $(7,8)$.
De tal manera que estos cambios se traducen en un incremento de la roncopatía del $14 \%$ en los 2 primeros trimestres, al $24 \%$ al final de embarazo (7,9-12); pudiendo estar asociado además a un aumento de los episodios de obstrucción de la vía aérea que incrementaría las complicaciones durante el embarazo.

En vista de que se ignora la magnitud de este problema en nuestra población, se decidió realizar un estudio de corte transversal, valiéndose de herramientas sencillas con el objetivo de determinar la frecuencia y distribución de la roncopatía durante el embarazo.

\section{MATERIALY MÉTODOS}

Se realizó un estudio transversal y descriptivo en el servicio de Obstetricia del Hospital Nacional Cayetano Heredia (HNCH), desde el $1^{\circ}$ de setiembre al 6 de octubre del 2004, con el fin de determinar la frecuencia y distribución de la roncopatía. El tamaño muestral se calculó utilizando un coeficiente de confidencialidad de 1,96 para un intervalo de confianza (IC) del 95\%, con un error absoluto calculado en 5\%. Es así que para una prevalencia reportada en la literatura del 24\% (9), el tamaño de la muestra fue de 280 pacientes.

Se incluyeron en el estudio a pacientes puérperas hospitalizadas en el servicio de obstetricia del HNCH y que hubieran firmado el consentimiento informado. El diagnóstico de hipertensión inducida por la gestación se definió en aquellas pacientes que presentaran una presión arterial (PA) sistólica mayor o igual a $140 \mathrm{~mm}$ $\mathrm{Hg}$ o una PA diastólica mayor o igual a $90 \mathrm{~mm} \mathrm{Hg}$ 
registrada en dos oportunidades entre el momento de su admisión al hospital y el puerperio inmediato (13). Se excluyeron a las pacientes cuya historia clínica no contaba con la hoja de control prenatal (CLAP), pacientes con edad gestacional menor a 30 semanas de embarazo, pacientes con patología no obstétrica concomitante (HIV, TBC, diabetes, etc.) o con embarazo gemelar, pacientes analfabetas, así como las pacientes que no completaron adecuadamente los cuestionarios.

Se emplearon tres cuestionarios para evaluar la presencia de somnolencia, roncopatía así como la calidad del sueño; además de aplicar una herramienta morfométrica.

Cuestionario de Roncopatía (CR): Cada paciente (con ayuda de su compañero de dormitorio) gradúa la frecuencia de ronquidos en las últimas semanas del embarazo en una escala de 1 a 5 . Se clasificó como roncadoras habituales a aquellas que respondieron 4 ó 5 , con una especificad del $84 \%$ y sensibilidad del 38\% $(10,14)$. Se preguntó además por la presencia de ronquidos antes del embarazo.

Escala de Somnolencia Epworth Modificado (ESE): Mide el grado de somnolencia diurna. En una escala de 0 a 3 se gradúa la posibilidad de quedarse dormida mientras se realizan 8 actividades propuestas en el cuestionario. Todo resultado igual o mayor a 10 fue considerado como somnolencia patológica (15-17).

Prueba de Calidad del Sueño (PCS): Consta de 34 preguntas sobre la sintomatología más común a los trastornos del sueño. De la pregunta 1 a la 9 identifica síntomas de SAOS; de la 10 a la 17 somnolencia; de la 18 a la 22 asma nocturna o reflujo gastroesofágico (RGE); de la 23 a la 28 síntomas de fibromialgia, síndrome de piernas inquietas y movimientos periódicos de los miembros; de la 29 a la 34 trastorno de adaptación, depresión o ansiedad; y las preguntas 11, 13 y 17 corresponden a síntomas de hipersomnia y narcolepsia. Este cuestionario ha sido utilizado por Vizcarra y col $(18,19)$.

Medida Morfométrica de Kushida (MMK): Esta prueba se basa en realizar mediciones en la boca y cuello de las pacientes, con un compás sin punta, el cual es llevado a una cinta métrica. Todas las medidas son incluidas en la siguiente fórmula matemática:

$(\mathrm{P} m m+(\mathrm{Mx} m m-\mathrm{Mn} \mathrm{mm})+3 \mathrm{x} \mathrm{OJ} m m)+3 \mathrm{x}$ [IMC $\left.\mathrm{kg} / \mathrm{m}^{2}-25\right] \times\left(\right.$ CC $\mathrm{cm} \div$ IMC $\left.\mathrm{kg} / \mathrm{m}^{2}\right)$
Donde: $\mathrm{P}=$ Altura palatina; $\mathrm{Mx}=$ Distancia entre los molares superiores; $\mathrm{Mn}=$ Distancia entre los molares inferiores; OJ = Distancia entre los incisivos superiores e inferiores; IMC = Índice de masa corporal; $\mathrm{CC}=$ Circunferencia del Cuello. Un resultado igual o mayor a 70 correlaciona con SAOS, con una sensibilidad del $97,6 \%$ y una especificidad del $100 \%$ en población caucásica mayor de 15 años y menor de 80 (20).

Los datos obtenidos se almacenaron en la base de datos Excel 2000 y se procesaron en los programas de EpiInfo v.3,3 y SPSS v.11,0. Mediante regresión logística se determinó la significancia de asociación entre variable dicotomicas. Se consideró estadísticamente significativo un $\mathrm{p}<0,05$. El estudio fue aprobado por el comité de ética de la Universidad Peruana Cayetano Heredia, así como el del HNCH y el Jefe del Departamento de Ginecología y Obstetricia del $\mathrm{HNCH}$.

\section{RESULTADOS}

De 304 pacientes hospitalizadas durante el periodo de estudio, se incluyeron 295. Se excluyeron tres pacientes con embarazo gemelar, dos portadoras del $\mathrm{VIH}$, tres con edad gestacional menor a 30 semanas, $\mathrm{y}$ una por llenar inadecuadamente los cuestionarios. Las características demográficas de nuestra población se encuentran representadas en la tabla 1.

Encontramos que el 44,7\% eran roncópatas y 10,5\% reunía criterios para ser roncadoras habituales. Sólo 22,4\% refirió roncar antes del embarazo. Adicionalmente $70(23,7 \%)$ pacientes reportaron tener somnolencia patológica, de éstas el 27,1\% eran roncadoras de novo y el $20 \%$ roncaban antes del embarazo. Sólo dos $(0,7 \%)$ pacientes tuvieron medidas morfométricas con valores alterados (Tabla 2). Por otro lado se halló que el 64,5\% de las pacientes roncadoras habituales tuvieron 4 o más respuestas afirmativas en la sección correspondiente a SAOS en la PCS (OR 3,34; IC 95\%: 1,53 - 7,27; p $0,001)$.

Se halló una frecuencia de hipertensión inducida por la gestación (HIG) del 13,6\% (40 pacientes). De estas el $40 \%$ eran roncadoras antes del embarazo y el 27,5\% eran roncadoras de novo. El 22,5\% de las pacientes con HIG eran roncadoras habituales; mientras que de las pacientes no hipertensas sólo 8,6\% eran roncadoras habituales. Así, una paciente roncadora habitual presentaría un riesgo 3 veces mayor de tener HIG (OR 3,07; IC 95\% 1,29 - 7,27; p 0,01). Las pacientes con 
Tabla 1. Características demográficas de las pacientes en estudio.

\begin{tabular}{|c|c|c|}
\hline & media \pm DS & n (\%) \\
\hline Edad & $25,97 \pm 6,71$ & \\
\hline Menor 25 años & & $146(49,5)$ \\
\hline Mayor 25 años & & $149(50,5)$ \\
\hline \multicolumn{3}{|l|}{ Estado Civil } \\
\hline Casada & & $54(18,3)$ \\
\hline Conviviente & & $189(64,1)$ \\
\hline Soltera & & $52(17,6)$ \\
\hline \multicolumn{3}{|l|}{ Grado de instrucción } \\
\hline Primaria & & $33(11,2)$ \\
\hline Secundaria & & $239(81,0)$ \\
\hline Superior & & $23(7,8)$ \\
\hline \multicolumn{3}{|l|}{ Raza } \\
\hline Mestiza & & $291(98,6)$ \\
\hline Negra & & $4(1,4)$ \\
\hline Número gestaciones & $2,26 \pm 1,32$ & \\
\hline Hijos a término & $1,03 \pm 1,16$ & \\
\hline Hijos prematuros & $0,02 \pm 0,15$ & \\
\hline Abortos & $0,22 \pm 0,50$ & \\
\hline Hijos vivos & $1,02 \pm 1,14$ & \\
\hline \multicolumn{3}{|l|}{ Número de Controles prenatales } \\
\hline$<4$ & & $47(15,9)$ \\
\hline$\geq 4$ & & $248(8,1)$ \\
\hline \multicolumn{3}{|l|}{ Ganancia de peso } \\
\hline$\leq 12 \mathrm{~kg}$ & & $178(62,5)$ \\
\hline$>12 \mathrm{~kg}$ & & $100(37,5)$ \\
\hline \multicolumn{3}{|c|}{ Indice de Masa Corporal al inicio del embarazo } \\
\hline$<25$ & & $198(69,2)$ \\
\hline$>25$ & & $88(30,8)$ \\
\hline Hipertensión inducida por la gestación & & $40(13,6)$ \\
\hline
\end{tabular}

una edad mayor o igual a 25 años tienen un riesgo 2 veces mayor de ser roncadoras habituales (OR 2,23; IC 95\%: 1,01 - 4,92; p 0,03), encontrándose una asociación similar al comparar las mayores de 30 años (OR 2,1; IC95\% 1,01-4,59; p 0,03). Se debe destacar que no se encontró asociación estadísticamente significativa cuando se comparó roncadoras habituales con IMC antes del embarazo, resultado de ESE y delta de peso durante la gestación.

\section{DISCUSIÓN}

El uso de una encuesta para el reporte de ronquidos es una herramienta útil para la detección SAOS. Bliwise y col. demostraron que el autoreporte de ronquidos correlaciona con los resultados objetivos de una polisomnografía, especialmente cuando se trata de roncadores habituales (21). Así, los sujetos roncadores habituales (gestantes o no) constituirían una población especialmente susceptible de presentar SAOS y cualquiera de sus complicaciones.
Tabla 2. Resultados de los cuestionarios $(\mathrm{n}=295)$.

\begin{tabular}{lcc}
\hline & $\mathbf{n}$ & $\%$ \\
\hline $\begin{array}{l}\text { Ronquidos antes del embarazo } \\
\text { Ronquidos en el te rcer trimes tre }\end{array}$ & 66 & 22,4 \\
$\quad$ Nunca & 163 & 55,3 \\
$\quad$ Raro & 55 & 18,6 \\
$\quad$ Algunas veces & 46 & 15,6 \\
$\quad$ Casi siempre & 13 & 4,4 \\
$\quad$ Siempre & 18 & 6,1 \\
Roncadora habitual (3 ${ }^{\circ}$ Trimestre) & 31 & 10,5 \\
Escala de somnole ncia de Epworth & & \\
$\quad \geq 10$ & 70 & 23,7 \\
$\quad<10$ & 225 & 76,3 \\
Me dida morfomé trica de Kushida & & \\
$\quad \geq 70$ & 2 & 0,7 \\
$\quad<70$ & 293 & 99,3 \\
\hline
\end{tabular}

En poblaciones europeas y norteamericanas la prevalencia de roncopatía registrada durante el tercer trimestre del embarazo varía de 40 a 49\% (9-11); y la prevalencia de roncadoras habituales se encuentra entre el 10 y el 14\% (10-12), resultados que correlacionan con los obtenidos en nuestro estudio. En la misma línea, la prevalencia de roncopatía previa al embarazo en estos estudios fue similar al 22,4\% reportado en esta muestra (9-12).

La similitud entre nuestros resultados y los de estudios previos, sugieren que la roncopatía durante el embarazo es un evento importante que se incrementa a lo largo de la gestación y que no dependería directamente de características étnicas, sino que más bien se trataría de una consecuencia de los cambios fisiológicos y anatómicos comunes al embarazo.

La ESE utilizada para diferenciar a las roncadoras simples (sin somnolencia diurna) de las roncadoras con posibles SAOS (con somnolencia diurna) $(15,16,22)$ es la misma que utilizara Loube y col., quien encontró un resultado promedio durante el embarazo de 9,2 superior al 6,85 encontrado en este estudio (9). Otros autores utilizando diferentes cuestionarios para medir somnolencia en el tercer trimestre, encontraron un $22 \%$ y $37 \%(9,11)$, mientras que nosotros encontramos sólo un $23,7 \%$. Probablemente, estos resultados disímiles radican en el uso de herramientas de medición distintas. Como en estudios previos, no encontramos ninguna relación entre el reporte de somnolencia durante el embarazo con los resultados de roncadoras habituales $(9,10)$. Así las quejas de fatiga y somnolencia diurna durante este periodo se deberían a factores intrínsecos del mismo y no a trastornos del sueño o ronquidos. 
Resaltando que en nuestro estudio no se buscan otras patologías del sueño como el síndrome de piernas inquietas, el cual se sabe que incrementa en el embarazo (12).

La medida morfométrica introducida por Kushida y col. no mostró ser fácilmente reproducible en el estudio (20). Una de las principales dificultades fue la ausencia de molares en un gran número de las pacientes que no permitió una medición precisa de la distancia intermolar. Aparentemente los resultados demostrarían que no sería aplicable en otro tipo de población diferente a la caucásica. Ajustes en la forma de medición, inicialmente propuesta, pudieran ser necesarios para su aplicación entre nuestros pacientes, enfatizando que el presente estudio no fue diseñado con ese objetivo.

Se sabe que la roncopatía es el principal síntoma y manifestación de SAOS y que esta aumenta con la edad $(1,2)$, bajo este contexto no resulta inusual haber encontrado que las pacientes de mayor edad tengan un riesgo incrementado de ser roncadoras habituales. De igual manera fue posible observar que un porcentaje mayor de pacientes roncadoras habituales presentaron 4 o más respuestas afirmativas en el área correspondiente a SAOS en la PCS. Esto podría indicar que las pacientes roncadoras habituales pudieran tener un riesgo incrementado (tres veces mayor) de tener SAOS; destacando que esta prueba no es diagnóstica pues para ello es necesario realizar una polisomnografía.

Se encontró que las roncadoras habituales presentaban un riesgo elevado de HIG. Sin embargo, es importante recalcar que el tamaño muestral no fue calculado para este fin. Como consecuencia obtenemos resultados con intervalos de confianza amplios. En las pacientes con preeclampsia se observa una variación del ritmo circadiano de la PA (23), así durante la noche la presión arterial no disminuye sino que incluso puede aumentar $(23,24,25)$. Se aprecia también una reducción del tiempo de sueño REM y de las etapas 3 y 4 del sueño no-REM, lo cual condiciona un aumento significativo de despertares durante la noche (26), asociado a una limitación en el flujo inspiratorio durante el sueño (27). En pacientes con preeclampsia la elevación de la PA podría estar asociada a periodos de apnea durante el sueño tal como sucede en pacientes no gestantes; encontrándose que un $30 \%$ a $40 \%$ de los pacientes con SAOS presentan HTA (3). Se sabe además que pacientes con alteraciones respiratorias moderadas a severas durante el sueño, presentan 2 a 3 veces más riesgo de sufrir HTA (28).
Así, la hipoxia y la hipercapnea producidas en los episodios de apnea, actúan sinérgicamente aumentando la descarga simpática en la musculatura del lecho vascular (5). El despertar posterior al episodio de apnea, aunado al aumento en el retorno venoso (por una elevada presión negativa intratorácica) contribuyen al aumento de la PA al final de un episodio de apnea $(5,28)$. Apneas obstructivas recurrentes producen un aumento crónico de la respuesta simpática, ocasionando HTA (5). Mecanismos similares se postulan para una asociación entre ronquidos y SAOS durante el embarazo con patologías obstétricas como la HIG y preeclampsia $(9,29,30)$, donde la descarga simpática actuaría sobre el lecho vascular placentario.

Los hallazgos de este estudio muestran un aumento en la frecuencia de ronquidos durante el embarazo que posiblemente radica en los cambios fisiológicos que tienen lugar en este periodo. Adicionalmente observamos que las pacientes roncadoras antes de la gestación presentaron casi el doble de frecuencia de HIG que las pacientes roncadoras de novo, estas últimas con una prevalencia similar a la población general. Se podría plantear la hipótesis que el efecto de la roncopatía crónica (posible SAOS) sobre el lecho vascular sería el que predispondría a presentar presiones elevadas durante la gestación, y no el efecto de la roncopatía de aparición reciente durante el embarazo.

\section{Correspondencia:}

Dr. Darwin Vizcarra Escobar.

Hypnos Instituto del Sueño. Clínica San Felipe. Av. Gregorio Escobedo 650, Jesús María. Lima, Perú. Fax: 511-6141105.

Correo electrónico: hypnos@clinicasanfelipe.com

\section{REFERENCIAS BIBLIOGRÁFICAS}

1. Naughton MT. Assessment and management of the patient presenting with snoring. Aust Fam Physician 2002; 31(11): 1-4.

2. Trotter MI, D' Souza AR, Morgan DW. Review articles: Simple snoring: current practice. J Laryngol Otol 2003; 117: 164-168.

3. Strollo PJ, Rogers RM. Current concepts: Obstructive sleep apnea. NEJM 1996; 334:99-104.

4. Flemons WW. Clinical Practice: Obstructive Sleep Apnea. NEJM 2002; 347:498-504.

5. García-Río F, Racionero M, Pino J, et al. Sleep apnea and hipertensión: The role of peripheral chemoreceptors and the sympathetic System. Chest 2000; 117:1417-1425. 
6. Malhotra A, White DP. Seminar: Obstructive sleep apnoea. Lancet 2002; 360: 237-245.

7. Edwards N, Middleton PG, Blyton DM, Sullivan CE. Sleep disordered breathing and pregnancy. Thorax 2002;57:555-558.

8. Santiago JR, Nolledo MS, Kinzler W, Santiago TV. Sleep and sleep disorders in pregnancy. Ann Intern Med 2001;134: 396-408.

9. Franklin KA, Holmgren PA, Jonsson F. Snoring, pregnancy-induced hypertension, and growth retardation of the fetus. Chest 2000; 117:137-41.

10. Loube DI, Poceta JS, Morales MC, Peacock MD, Mitler MM. Self reported snoring in pregnancy: Association with fetal outcome. Chest 1996; 109(4): 885-889.

11. Guilleminault C, Querra-Salva MA, Chowdhuri S, Poyares D. Normal pregnancy, daytime sleeping, snoring and blood pressure. Sleep Medicine 2000; 289297.

12. Hedman C, Pohjasvaara T, Tolonen U, Suhonen-Malm AS, Myllyla VV. Effects of pregnancy on mother's sleep. Sleep Med 2002; 3: 37-42.

13. Sibai BM. Diagnosis and management of gestational hypertension and preeclampsia. Obstet Gynecol 2003; 102(1): 181-192.

14. Lindberg E, Elmasry A, Janson C, Gislason T. Reported snoring: Does validity differ by age? J Sleep Res 2000; 9:197-200.

15. Murray WJ. A new method for measuring daytime sleepiness: The Epworth Sleepiness Scale. Sleep 1991; 14(6): 540-545.

16. Murray WJ. Reliability and factor analysis of the Epworth sleepiness scale. Sleep 1992; 15(4): 376-381.

17. Castro J, Vizcarra D, Alvarez J. Somnolencia diurna y síndrome apnea hipopnea del sueño. Rev Soc Peruana Med Int 2003; 16(2): 74-83.

18. Vizcarra D. Ronchopaty and chronic mountain sickness. Abstract. Philadelphia, Pennsylvania: Associated Professional Sleep Societies $18^{\text {th }}$ Annual Meeting, June 5-10, 2004.

19. Rey De Castro J, Vizcarra D. Frecuencia de síntomas del síndrome apnea hipopnea del sueño e insomnio en médicos de una clínica privada peruana. Rev Med Hered 2003; 14(2): 53-58.

20. Kushida CA, Efron B, Guilleminault C. A predictive morphometric model for the obstructive sleep apnea syndrome. Ann Intern Med 1997; 127:581-4.

21. Bliwise DL, Nekich JC, Dement WC. Relative validity of self-reporting snoring as symptoms of sleep apnea on a sleep clinic population. Chest 1991; 99:600-08.

22. Hoffstein V, Szalai P. Predictive value of clinical features in diagnosing obstructive sleep apnea. Sleep 1993; 16(2): 118-122.

23. Oney T, Meyer-Sabellek W. Variability of arterial blood pressure in normal and hypertensive pregnancy. J Hypertens Suppl 1990; 8(6):S77-81.

24. Sawyer MM, Lipshitz J, Anderson GD, Dilts PV Jr, Halperin L. Diurnal and short-term variation of blood pressure: Comparison of preeclamptic, chronic hypertension and normotensive patients. Obstet Gynecol 1981; 58(3):291-296.

25. Brown MA, Davis GK, McHugh L. The prevalence and clinical significance of nocturnal hypertension in pregnancy. J Hypertens 2001; 19(8):1437-1444.

26. Edwards N, Blyton CM, Kesby GJ, Wilcox I, Sullivan CE. Preeclampsia is associated with marked alterations in sleep architecture. Sleep 2000; 23(5): 619-625.

27. Connolly G, Razak AR, Hayanga A, Russell A, McKenna P, McNicholas WT. Inspiratory flow limitation during sleep in preeclampsia: comparison with normal pregnant and nonpregnant women. Eur Respir J 2001; 18:672-6.

28. Peppard PE, Young T, Palta M, Skatrud J. Prospective study of the association between sleep-disordered breathing and hypertension. NEJM 2000; 342:1378-84.

29. Edwards N, Blyton DM, Kirjavainen TT, Sullivan CE. Hemodynamic responses to obstructive respiratory events during sleep are augmented in women with preeclampsia. Am J Hypertens 2001; 14(11): 1090-1095.

30. Edwards N, Blyton DM, Kirjavainen T, Kesby GJ, Sullivan CE. Nasal continuous positive airway pressure reduces sleep-induced blood pressure increments in preeclampsia. Am J Respir Crit Care Med 2000; 162(1):252-257. 